\title{
CHARACTERIZATION AND AUTOMATIC COUNTING OF F.I.S.H. SIGNALS IN 3-D TISSUE IMAGES
}

\author{
UMESH ADIGA PS ${ }^{1}$, SAMANTHA JL KNIGHT ${ }^{2}$ AND CHAUDHURI BB ${ }^{3}$ \\ ${ }^{1}$ Wellcome Trust Centre for Human Genetics, University of Oxford, Roosevelt Drive, Headington, Oxford, \\ OX3 7BN, UK, ${ }^{2}$ Institute of Molecular Medicine, Oxford University, ${ }^{3}$ CVPR Unit, Indian Statistical Institute, \\ 203, BT Road, Calcutta-35, India \\ e-mail: umesh.adiga@well.ox.ac.uk, sknight@molbiol.ox.ac.uk, bbc@isical.ac.in
}

(Accepted March 12, 2001)

\begin{abstract}
The evaluation of malignancy-related features often helps to determine the prognoses for patients with carcinomas. One technique, which is becoming increasingly important for assessing such prognostic features is that of Fluorescence in situ Hybridization (FISH). By counting the number of FISH signals in a stack of 2$\mathrm{D}$ images of a tumor (which together constitute the 3-D image volume), it is possible to determine whether there has been any loss or gain of the target DNA sequences and thereby evaluate the stage of the disease. However, visual counting of the FISH signals in this way is a tedious, fatiguing and time-consuming task. Therefore, we have developed an automated system for the quantitative evaluation of FISH signals. We present and discuss the implementation of an image processing module that segments, characterizes and counts the FISH signals in 3-D images of thick prostate tumor tissue specimens. Possible errors in the automatic counting of signals are listed and ways to circumvent these errors are described. We define a feature vector for a FISH signal and describe how we have used the weighted feature vector to segment specific signals from noise artifacts. In addition, we present a method, which allows overlapping FISH signals to be distinguished by fitting a local Gaussian model around the intensity profile and studying the feature vector of each model. Our complete image processing module overcomes the problems of manual counting of FISH signals in 3-D images of tumor specimens, thereby providing improved diagnostic and prognostic capability in qualitative diagnostic pathology.
\end{abstract}

Keywords: characterization, counting, features, FISH signal.

\section{INTRODUCTION}

The evaluation of malignancy-related features often helps to determine the prognoses for patients with carcinomas. One technique, which is becoming increasingly important for assessing prognostic features is that of Fluorescence in situ Hybridization (FISH). FISH involves the fluorescent tagging of selected DNA sequences so that they may be visualized under the microscope. The DNA sequences may be chosen either to detect specific abnormalities or to facilitate the process of identification and quantification of numerical and structural chromosomal abnormalities. Several researchers have shown the reliability of the technique for the evaluation of numerical chromosome aberrations (Aubele et al., 1996). By counting the number of FISH signals it is possible to determine whether there has been any loss or gain of the target DNA sequence. Large variations in the FISH count have been shown to be associated with a high histological grading (Baretton et al., 1994) and an advanced stage of disease (Henke et al., 1994).
Indeed, several studies (Babu et al., 1990; Johnson and Nogueira, 1981), have shown that trisomy of chromosome $\# 7$ is found in malignant and nonmalignant tumors of lung, kidney, brain, as well as in prostate gland. Thus, the quantitative evaluation of the loss or gain in DNA is useful in qualitative diagnostic pathology.

However, one of the disadvantages in quantitative evaluation of the FISH signals based on the conventional microscopy images is the considerable number of nuclei sliced during the sectioning and/or thin specimen preparation. Such truncated nuclei do not represent the complete chromatin of the cell nuclei and lead to the incorrect evaluation of the FISH signal count. To guarantee a reproducible, unbiased signal count per nucleus and realistic feature values, a complete cell nucleus has to be inspected and analyzed in three dimensions (Aubele et al., 1996). In addition, to estimate the distribution of FISH signals per cell, a large number of cells have to be analyzed, especially when the frequency of aberrant cells is low. 
Visual counting of the FISH signal in a 3-D stack of images is a tedious, fatiguing and time-consuming task. To facilitate the counting, one has to display a gallery of all the 2-D images, which together constitutes the image volume. If the tissue architecture shows large density of cells, then, the amount of work increases proportionally. This is because, a gallery of images of a box around each cell of interest has to be displayed for clear presentation. Fig. 1, shows a gallery of a part of the image depicting a particular cell, which can be used for visual counting of the FISH signals. Even using this approach, erroneous counting is likely since the visual correlation of the same signal in different slices is difficult. Therefore, for improved diagnostic and prognostic ability, it would be extremely valuable to have an automated system for the quantitative evaluation of FISH signals. Despite this, little work has been done to date on automating such processes. This is mainly due to the involvement of difficult tasks such as 3-D segmentation of the tissue to mark the region of interest in the FISH signal channel, assigning the FISH signal membership, distinguishing noisy clusters from the genuine FISH signals and the automated counting. The algorithms for automated FISH signal counting must be accurate and the number of false negatives must be as low as possible. Conversely, the algorithm must be fast and efficient. In the image volume, FISH signals appear like a tiny objects consisting of high intensity voxels extending to several image slices and they are located entirely within the cell nucleus. Fig. 2a, shows the surface rendered display of the FISH signals while Fig. 2b, shows one of the image slices of the FISH signal channel.
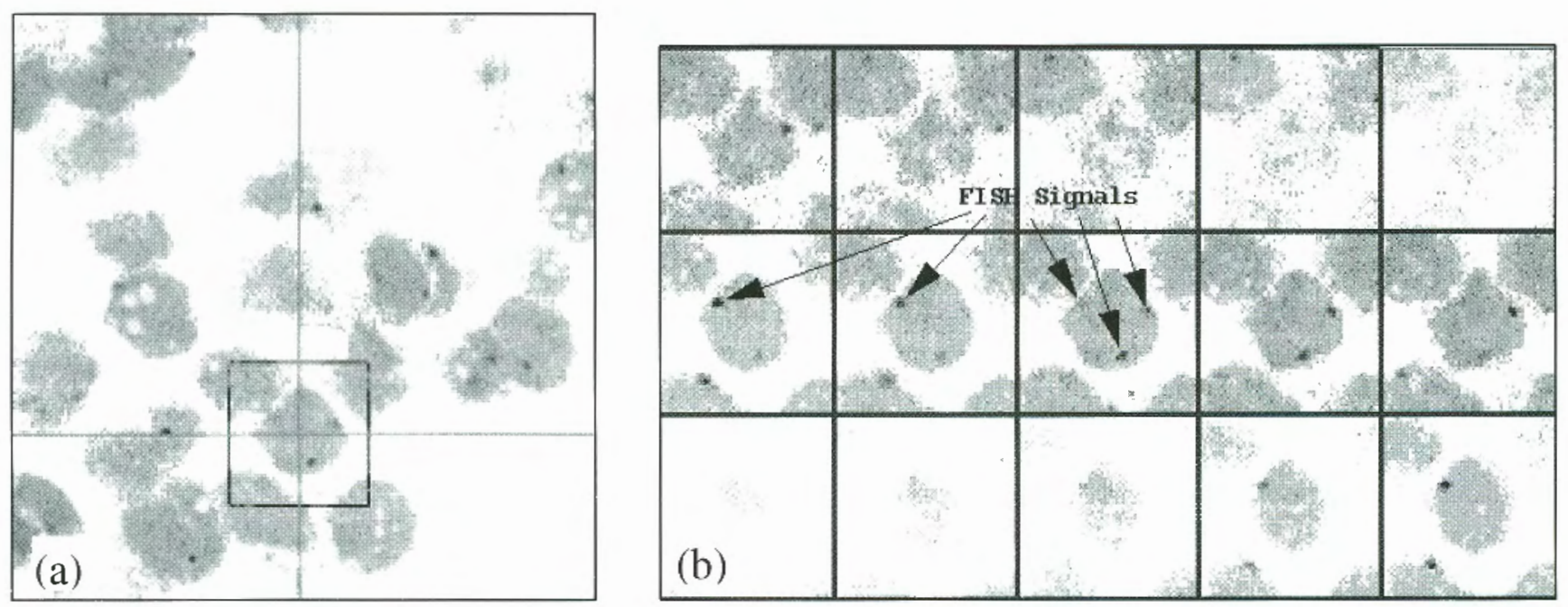

Fig. 1. Image display for visual (manual) counting of FISH signals in a cell (256×256 pixel image). (a) Selection of a cell of interest by drawing a box around it. (b) A cell of interest is displayed as a gallery of images for visual FISH signal counting. (the box image is magnified by factor 2 and displayed).

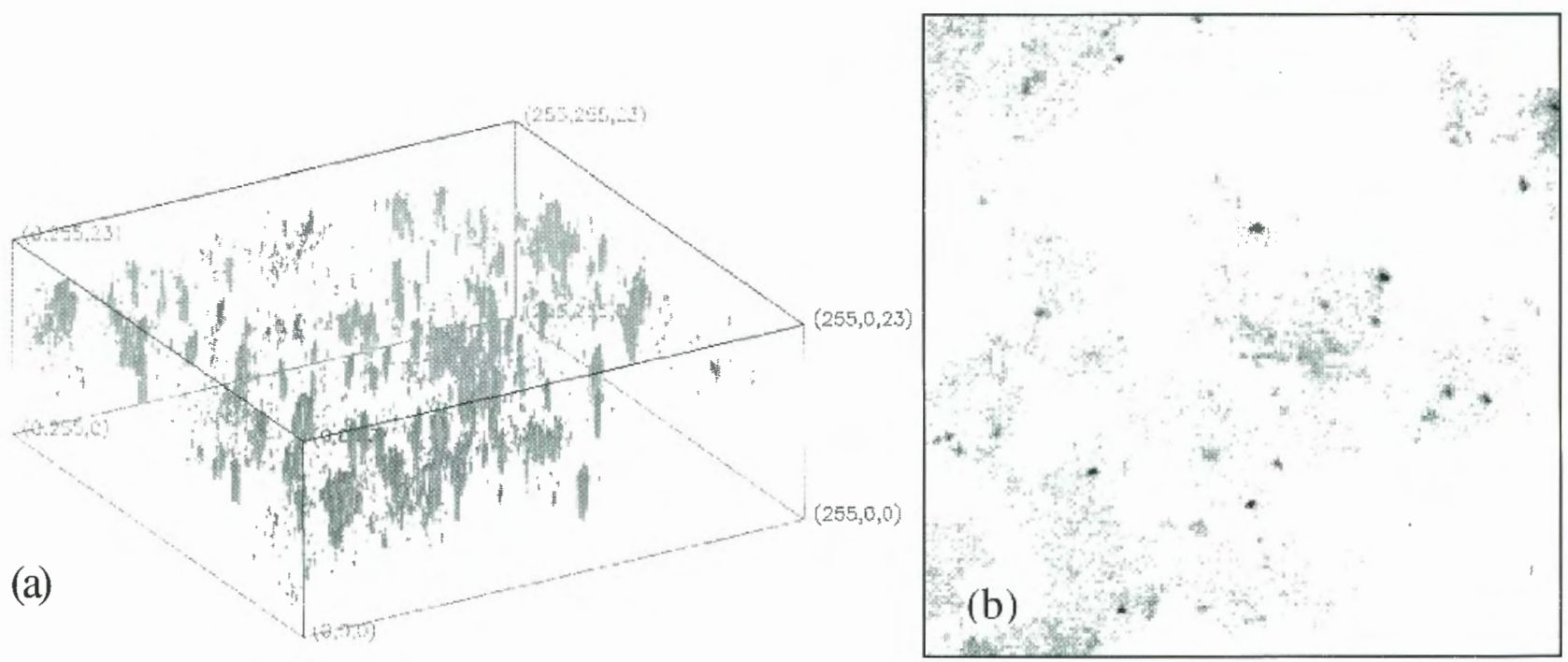

Fig. 2. FISH Signal Channel. (a) Surface rendered display of the FISH signal channel, (b) Middle optical section (256×256 pixel image) of the FISH signal channel of the volumetric image. 
We have implemented and/or developed several 3-D tissue segmentation methods to separate the cells in a cluster and label them. One or more of these methods can be used sequentially depending on the complexity of tissue architecture as shown in image. The 3-D tissue segmentation techniques we have developed and/or implemented include 1) the layered segmentation method (Adiga and Chaudhuri, 1998a) 2) active surfaces (Adiga and Chaudhuri, 2000a) and 3) region-based segmentation techniques such as seeded volume growing, successive pealing and thickening, 3-D watershed with rule based merging (Adiga and Chaudhuri, 2000b, 2000c) and other integrated techniques (Adiga, 2000).

\section{MATERIAL}

The tumor specimens were prepared by pathologists at the GSF, Munich. These routinely processed formalin-fixed and paraffin embedded tissue specimens from radical-prostatectomies of several patients with prostatic adenocarcinoma were used to assess whether 3-D evaluation would be desirable to facilitate the counting of FISH signals present in each cell (Aubele et al., 1996). Details of specimen preparation and the protocols used have been described previously (Rodenacker et al., 1997; Aubele et al., 1996).

The software we describe was implemented on an SGI-IRIX5.2 machine using Interactive Data Language (IDL) and C. Evaluations were done on about 150 data sets. The number of cells per data set and number of FISH signals per data set varied from one volumetric image to another. The acquisition of the images is described below.

Set-up Features for Image Acquisition: Fluorescence images were scanned using a Confocal Laser Scanning Microscope (CLSM) Zeiss LSM 410. Essential set-up features for the acquisition of FISH signals in tissue sections were as follows: Lens Zeiss PNF $100 \times$, numerical aperture 1.3 , zoom $=2$, realized by the scanning unit. The scanned field of $62.5 \mu \mathrm{m} \times 62.5 \mu \mathrm{m}$ was sampled to $256 \times 256$ pixels giving a pixel size of $0.25 \mu \mathrm{m}^{2}$ in $\mathrm{x}$ and $\mathrm{y}$ directions. Excitation laser lines were selected according to the fluorochromes used. Both propidium iodide (PI), used as a DNA counter-stain, and Fluorescein isothiocyanate (FITC) used to tag the specific DNA target sequences, were excited by the Argon line 488 $n \mathrm{~m}$. The emissions of both PI and FITC were measured simultaneously in two separate channels using a band-pass $515-565 \mathrm{~nm}$ for FITC and a lowpass LP 590 for the PI channel. The axial distance selected between two subsequent confocal images depends on a further evaluation strategy. If a spatial isotropic representation of the 3-D data is intended, the axial distance is identical to the lateral distance of the pixels, i.e. $0.25 \mu \mathrm{m}$. For a $16 \mu \mathrm{m}$ thick section this results in a sequence of 64 scanned images. However, if the sampling theorem only needs to be an approximation, then approximately $0.5 \mu \mathrm{m}$ axial size can be obtained. The fluorescence emission of FITC is associated with the green channel whereas the PI signals (depicting the tissue architecture) are associated with the red channel of a RGB color image. The image data are stored on disk in TIFF format.

\section{EVALUATION OF FISH SIGNALS}

Visual counting of FISH signals is a difficult, time consuming and cumbersome process. Before attempting any automated method based on heuristics or otherwise, it is important to define the morphological properties and parameters of a FISH signal. We define a fluorescence signal as a spatial spot with parameters like size, shape, intensity, etc., within a specified range. The parameters of each signal can be obtained in two steps namely (i) detection and (ii) estimation. In case of overlapping signals, it is quite difficult to distinguish the signals if the intensity peaks of the two overlapped signals are very close to each other. To address this, we have incorporated a technique to characterize the overlapped signals by fitting a Gaussian model to the intensity profile of the overlapped signal. The features signal under each Gaussian profile is separately estimated to decide the validity of the signal.

The simplest algorithm for detecting a FISH signal is to dissect its distinguishing property from the background. The usual approach is to suppress noise by filtering the image and to enhance the signal by a suitable method. Then the features of the signals are estimated to distinguish the actual FISH signal from the artifact. The region of interest for counting the FISH signals is only within the cell nuclei. Hence all the voxels that fall outside the cell are discarded. For this purpose the segmented and labeled tissue image is superposed virtually on the signal channel by one to one mapping. Thus the non-cellular regions, regions belonging to truncated cells, and highly deformed cells, etc., are screened out.

\section{POSSIBLE ERRORS IN SIGNAL IDENTIFICATION}

The FISH signal channel is usually corrupted by hardware noise or error due to the specimen fixation 
process. Some of the errors caused due to noise or other means in the FISH signal channel are, 1. split FISH signals, 2. overlapping FISH signals, 3. missed FISH signals, 4. false FISH signals, 5 . out of focus FISH signals, 6. debris, 7. loss of FISH signal due to over segmentation, 8. increased FISH signal count due to improper segmentation, 9. error in defining the FISH signal, 10. error due to ambiguity about FISH signal located near the boundary, 11. error due to FISH signal located on the overlapped or touching portion of the cell nuclei.

1. Split FISH signal error occurs when there is only one signal but two are counted. This error occurs when the signal lies on the common boundary of the touching cells and so is counted as a FISH signal in both the cells. This error can be avoided as follows. If the signal intensity peak and at least $40 \%$ of the FISH signature is in one cell then the signal is counted as belonging to that particular cell.

2. Overlapping FISH signals error occurs when there are two or more FISH signals, but only one is counted. This results in under-estimation of the signal density. This error may occur due to overlapping signals in the specimen itself or when the signal channel is smoothed and thresholded during detection phase. If the FISH signals are located close to one another they may join together during smoothing. If the resolution of the image stack is poor it may also cause two closely located FISH signals one below the other to be counted as only one. Characterization of the overlapping signals is a difficult problem. If the overlapping signals show two distinct intensity peaks in the intensity profile, then it is possible to match the signal model to each intensity peak and decide whether two signals are really overlapping. In section 4, 'Detection and Counting of Fish Signals', we have explained the method to detect and count overlapping FISH signals.

3. Missed FISH signal occurs when FISH signals that are present are not counted. This error is due to poor segmentation of the cells in the tissue, causing only partial cell chromatin to be identified as the cell, while the rest is considered as background. Due to uneven illumination of the specimen and attenuation of light intensity along the depth, some portion of the cell may not be recognized during thresholding. Any signal present in those parts is deleted in the signal channel too, as we are not considering the voxels outside the cell region. Also, the attenuation of the light intensity and the photobleaching of the fluorescent labeled specimen may cause the deletion of some signals in the specimen. Wrong feature calculation of the signals during segmentation and identification process may also cause the missed FISH signal error.

4. False FISH signal error occurs when signals that are not true FISH signals are counted. This is one of the main causes for erroneous signal count. Noise, artifacts due to improper dye application, specimen damage, etc., get prominently reflected in the FISH signal channel. Sometimes, it is not even possible to distinguish them visually from the real FISH signal. Proper specimen preparation, noise reduction, selection of the right features, etc., can reduce the error caused by a false FISH signal.

5. Out-of-focus FISH signal error is caused by the presence of out of focus information in the FISH signal channel or out of focus cells in which FISH signals can hardly be seen. Since the confocal imaging reduces the out-of-focus information considerably, this error is less predominant. In the case of wide-field microscopy and other imaging techniques, the blurred FISH signal may not satisfy the criteria set for identifying the FISH signal causing errors. This error can be reduced by deblurring the image using the Wiener filter or other methods (Roysam et al, 1995).

6. Debris error occurs when the hybridization procedure has been sub optimal. Examples of this would be extraneous, fluorescing materials in the specimen and the presence of air bubbles, both of which would be reflected in the image and lead to errors in FISH signal counting. Fortunately, most of the debris can be removed by simple size filtering and proper featuring of the signals.

7. Over-segmentation of the tissue causes a major error in the evaluation of FISH signals. If the same object is divided into several objects with different labels, it causes underestimation of the FISH signal count per cell nucleus. Similarly, undersegmentation (where more than one cell is given the same label) causes overevaluation of FISH signal count. Any error in marking the cell boundary causes an error in the FISH count when the signal lies very close to the boundary. If the signal lies on the touching or overlapped cell boundary, an ambiguity arises about the membership of the FISH signal. As mentioned earlier, the signals lying on the common boundary of the cells can be assigned to a 
particular cell by checking the location intensity peak in the signal profile and the size of the signal signature in each of the cells.

8. Wrong selection of FISH signal features causes major errors. In this case, the FISH signal channel is contaminated by various types of noise. No noise reduction technique completely removes the artifacts without affecting the signal considerably. Hence it is very important to distinguish the FISH signals from the noise artifacts, based on proper features. Wrong selection of features results in either counting the group of noisy voxels as FISH signals, or rejecting a weak FISH signal as a noise artifact.

\section{FEATURE SELECTION FOR F.I.S.H. SIGNALS}

It is possible to choose various image features of the FISH signals to identify them and segment them from the noise artifact. As there is neither definite shape, size, orientation nor location of the signal within the cell nuclei, one first has to undertake a supervised feature selection on a large number of data sets before finalizing the list of FISH signal features and the range of values one should most probably take. We have undertaken the visual identification and interactive segmentation of the FISH signals of over fifty image data sets of thick prostate tissue specimens. This manual analysis has allowed us to select some main features and value ranges, which can be used to identify signals in testing data sets. Some of the FISH signal features, which have been identified in this way are 1 . volume of the FISH Signal $V$., 2. relative volume defined as the ratio of the size of the FISH signal to the average, size of all the FISH signals identified within the corresponding cell nucleus $R$., 3. average intensity of the FISH signal $I_{\text {avg., }}$ 4. relative intensity defined as the ratio of the average intensity of the FISH signal and the average intensity of all the FISH signals within the corresponding cell nucleus $I_{\mathrm{r}}$, 5. total fluorescent intensity $I_{\text {tot., }}, 6$. location of the FISH signal s., 7. shape (three-dimensional) of the FISH signal $\gamma, 8$. local background gray level $B$.

Thus, the parameter vector can be written as $\vec{P}=\left[V, R, I_{\text {avg }}, I_{r}, I_{\text {tot }}, s, \gamma, B\right]^{T}$. For the data used as test sets, the FISH signals have first been visually identified with the help of a pathologist. The parameters defining these FISH signals are then calculated to find lower and upper limits for these features. Thresholds for the features such as relative size and relative intensity are also set experimentally. Based on the experimental results on the test data sets we have defined the range and the average value for each of the parameters in vector P. Before measuring the features of the signals, the noise associated with the channel should be reduced and the signals have to be enhanced to distinguish them from the background. We have used a hybrid median filter, Gaussian smoothing and point finders such as top-hat filters to reduce the noise and enhance the FISH signals. Size and shape heuristics along with the other features mentioned above are then used to identify and segment the FISH signals.

\section{NOISE REDUCTION AND SIGNAL ENHANCEMENT}

Median Filtering: In the first step, we have used a two-dimensional median filter on each image slice. A simple median filter replaces the input pixel by the median of the pixels contained in a small window around the pixel. We have used a $3 \times 3$ window $\mathrm{W}$ for this purpose. If $v(x, y)$ is the filtered pixel value then, $v(x, y)=\operatorname{median}\{u(x-m, y-n)\}$, where $(m, n) \in W$. This filter removes isolated pixels and the binary noise in the signal channel. We have not used threedimensional median filtering since the larger window would increase the computation load.

Gaussian Smoothing: The next step is smoothing. FISH signals are localized features. Simple spatial averaging would not only blur the signal but would also increase the chances of connecting two closely located signals. Therefore we used Gaussian smoothing. This gives less importance to far away voxels and so the distortion of FISH signals will be minimized by Gaussian filtering. The 3-D Gaussian function is given as $G[i, j, k]=e^{\left\{-\left(i^{2}+j^{2}+k^{2}\right) /\left(2 \sigma^{2}\right)\right\}}$. The degree of smoothing is characterized by $\sigma$. We have used a symmetrical Gaussian filter with $\sigma^{2}=2$. Because of Gaussian smoothing, weak noisy signals and isolated voxels are smoothed out. Fig. 5b shows a representative image slice of the FISH signal channel after Gaussian smoothing. As can be seen, there is some degree of blurring of the FISH signal after smoothing. Top-hat filtering is used to enhance a smooth FISH signal by comparing it with a local background gray value. In our image processing module, we have extended the two-dimensional tophat filter to a three-dimensional filter. This is done because the FISH signals are essentially threedimensional in nature. The three-dimensional top-hat filtering technique is explained below. 
Top-hat Filtering: The ideal top-hat filter is a point finder. It consists of a flat disc that rests on a surface and a central crown of smaller diameter. This filter is centered on each pixel in the image, with the brim resting on the surface as shown in Fig. 3. Any signal that 'sticks up' through the crown of the hat is considered to be a probable FISH signal and is brightened up to a higher level. The size of the central crown is defined by the smaller of the two neighborhood regions as shown in the Fig. 4. The larger region represents the local background, which the point of interest must exceed in brightness. The top-hat method finds the maximum brightness in the larger surrounding region and subtracts it from the brightest point in the interior region. If the difference exceeds the previously defined threshold, which is located at the height of the crown of the top-hat filter, then that point in central crown is considered as a signal.

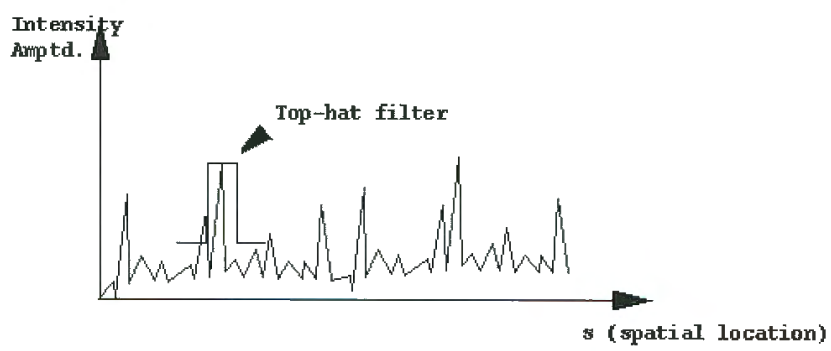

Fig. 3. Simple example of top-hat operation on onedimensional sequence of signals.
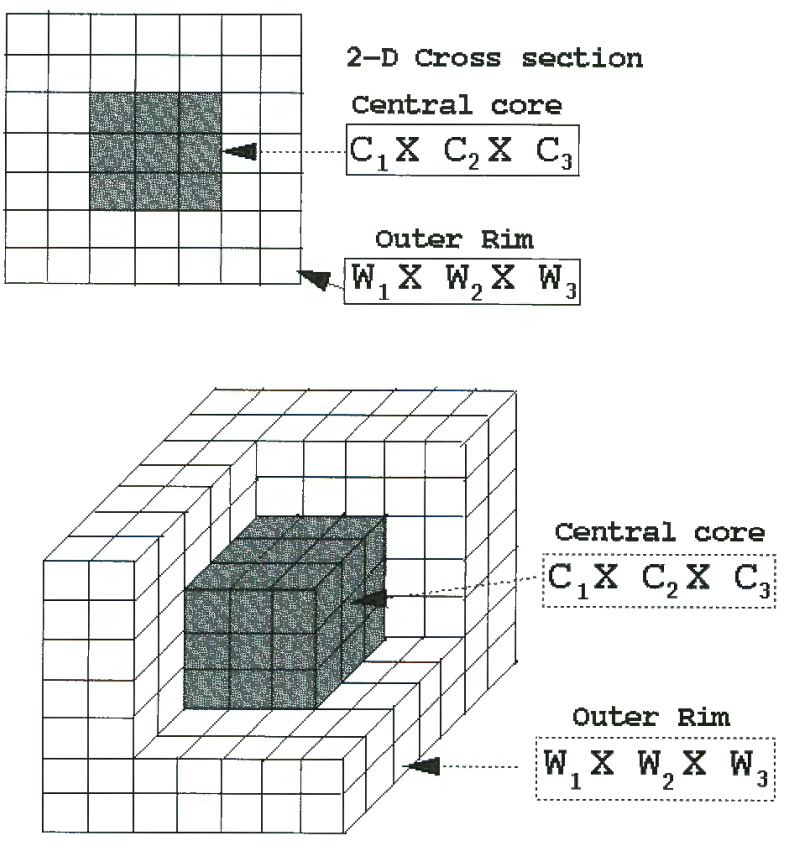

3-D Top-hat filter

Fig. 4. Three-dimensional top-hat filter.
The 3D top-hat filter consists of $C_{1} \times C_{2} \times C_{3}$ core voxels in a cube of the size $W_{1} \times W_{2} \times W_{3}$ window, where $W_{i}>C_{i}$ for all $i=1,2,3$. Fig. 4 shows diagrammatically the $3 \mathrm{D}$ top-hat filter we have used with $W_{1}=W_{2}=W_{3}=5$ and $C_{1}=C_{2}=C_{3}=3$ This filter is centered on each voxel in the image. The tophat method finds the maximum brightness in the larger surrounding region and subtracts that from the brightest point in the interior core region. If the difference exceeds some pre-defined threshold, then that point in central crown is considered as FISH signal and is enhanced. The threshold is chosen as $\mu+k$ where $\mu$ is the average gray value of the window $W_{1} \times W_{2} \times W_{3}$ and $k$ is an experimentally determined constant. Fig. 5c, shows a single representative image slice of the FISH signal channel after top-hat filtering.

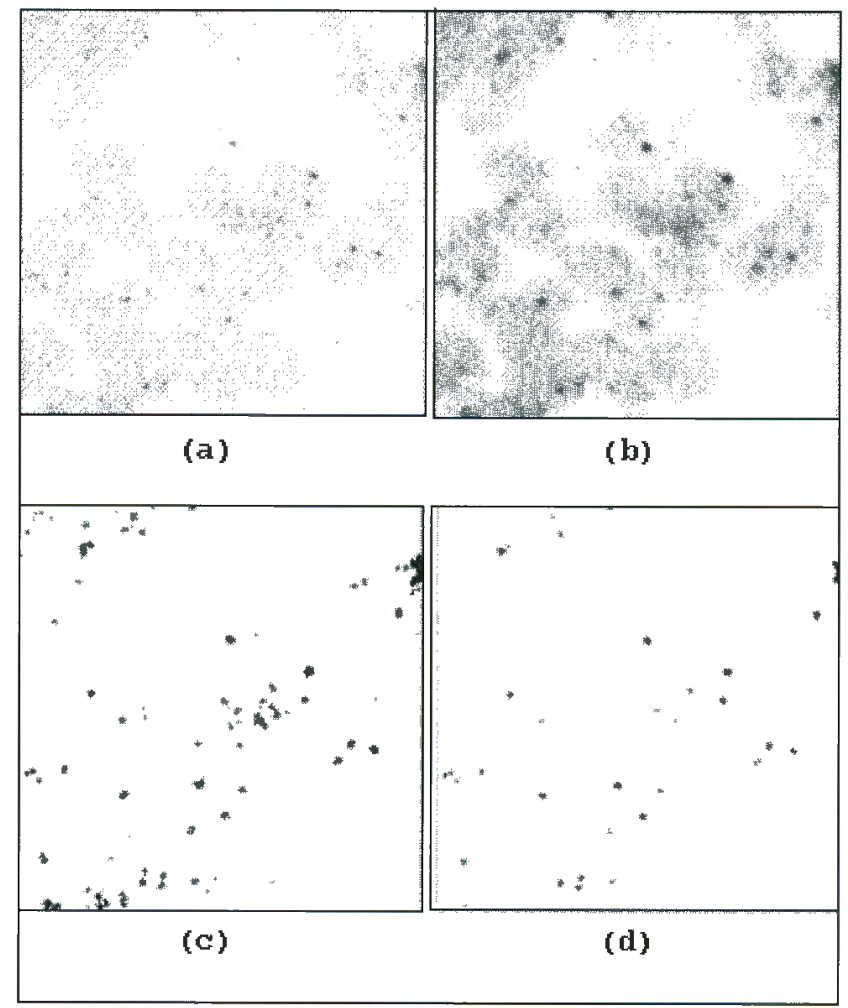

Fig. 5. Result of different steps of noise reduction shown over a single representative image of size. 256×256. (a) Original image slice (b) After Gaussian smoothing (c) After top-hat filtering (d) After Size and Shape filtering.

Size and Shape Filtering: When there is a cluster of noisy voxels with relatively high intensity, smoothing and thresholding may result in making the noisy cluster into a connected component. This can cause error in the automatic counting of FISH signals. Also, the air bubbles and improper use of fluorescent materials are reflected as relatively large objects in 
FISH signal channel. Such noises and artifacts are removed by size and shape heuristics. The maximum and minimum size of the FISH signal is defined. The objects, which do not fall within the size criteria are considered as noise artifacts and removed. The maximum size range is kept as three times the average size of the signal, so that overlapped signals are not rejected before being analyzed. Though FISH signals do not have any definite shape, it is observed that these signals are also three dimensional in nature. Hence, only those signals which are within the predefined size range and occupy at least two consecutive slices in the image stack is considered as valid signal. Fig. 5d shows the effect of noise filtering using size and shape filtering.

\section{DETECTION AND COUNTING OF F.I.S.H. SIGNALS}

A reasonable method to detect FISH signals and to determine their parameters should have the following properties.

- The method should be translation, scaling and rotation invariant.

- The method should be able to detect the range of parameters of the signal.

- The accuracy by which the parameters are determined must be as accurate as the level of noise permits. Thus, the method should degrade smoothly as the level of noise monotonically increases.

- For further analysis, the method should yield a measure of certainty for each signal by describing how well it resembles an ideal FISH signal.

- The method should require a minimal number of user-defined parameters. Also, it should be as generally applicable as possible and be efficient in computer time and memory.

A simple algorithm for detecting the signals, which satisfies the above mentioned conditions, is to threshold the image at an appropriate level and characterize the signal by using its property to distinguish it from background. All the voxels, which exceeds a particular threshold are examined for whether they satisfy the range of feature values present in the feature vector $\mathrm{P}$. Before calculating the feature vector the objects are labeled using 3-D component labeling algorithm. Each labeled object is considered as a possible FISH signal.

The 3-D component labeling based on simple region growing is used to label the possible FISH signals within a specified region. The signal channel of the multi-spectral, volumetric image is scanned from top left in a raster pattern. Whenever a voxel of an object (possible FISH signal) is found, all the voxels connected to it are given a unique label. The scanning is continued from the point where it was left till another voxel, which is not already labeled but belongs to a signal is found. In this way a complete image is scanned and all the voxels belonging to an object are given a unique label. The result of labeling also gives the number of objects that may constitute a FISH signal in the cell nucleus. The process is repeated for all the cells and the possible FISH signals per cell nucleus are documented for further analysis.

$$
\text { Let } W=\left[w_{V}, w_{R}, w_{I_{\text {avg }}}, w_{I_{r}}, w_{I_{\text {zoo }}}, w_{s}, w_{\gamma}, w_{B}\right] \text { be }
$$

the weights assigned to different features of the FISH signals described in the feature vector respectively. If

$$
\vec{P}=\left[V, R, I_{a v g}, I_{r}, I_{t o t}, s, \gamma, B\right]^{T}
$$

is the feature vector defining the FISH signal, and the vector function defining the decision based on each feature

$\left.\left.\overrightarrow{d(\vec{P})}=\left[\overrightarrow{d(S)}, \overrightarrow{d(R)}, \overrightarrow{d\left(I_{\text {avg }}\right)}, \overrightarrow{d\left(I_{r}\right.}\right), \overrightarrow{d\left(I_{\text {tot }}\right.}\right) \overrightarrow{d(s)}, \overrightarrow{d(\gamma)}, \overrightarrow{d(B)}\right]^{T}$ then, an object in the image space is considered as FISH signal if the weighted combination of the decisions taken over individual features, is above a pre-defined threshold. The elements in the decision vector take the value 0 or 1 . If the value of the feature is within a predefined range, the $\overrightarrow{d(.)}$ elements take a value of 1 , otherwise the $\overrightarrow{d(.)}$ are 0 . All the thresholds are chosen experimentally using test sets. Features of each FISH signal are estimated and the range of the feature value from the minimum-to-maximum value found in the design set is considered as the threshold range.

Let us represent the decision as a linear combination of corresponding weight and decision vector elements, $\vec{F}_{i}=\sum_{j} W_{j} \cdot d_{j}\left(P_{j}\right)$, to be the weighted decision vector of the possible FISH signal under inspection. If $\vec{F}$ is weighted feature vector of an ideal FISH signal, then the match error between the ideal FISH signal and the signal $i$ under inspection can be defined as

$$
e_{i}=|\vec{F}-\vec{F}|^{2}
$$


If this error is below an experimentally defined threshold range, then the signal is considered as authentic and counted. If the error is above the threshold, then the image element (connected component which constitutes a spot or FISH signal) may be an overlapped FISH signal or is a noise artifact due to debris, etc..

Automatic detection of the overlapped signals is done by inspecting and characterizing the intensity profiles and the feature vector of separated signals. The signals, which do not conform to the matching error are subject to a second stage of analysis. Here, the signals are tested for overlapping error. If the size of the signals are below the lower threshold of the size feature range, such signals are discarded as artifacts. If they are above the maximum size of the size feature range and intensity features such as $I_{\text {avg }}, I_{\text {tot }}, I_{r}$ are also above the average feature range, then overlapping of two or more FISH signals is suspected. We have used a Gaussian model fitting technique to resolve the overlapped signals and to find the parameters of the feature vector of such signals (Noordmans and Smeulders, 1998).

First, the connected (labeled) region constituting a spot is searched for multiple intensity peaks. If multiple intensity peaks of approximately identical magnitude are present, then a local Gaussian model of the signal is extracted for each intensity peak. Fig. 7 shows a schematic diagram in 1-D depicting the process of fitting the Gaussian model to each intensity peak present in the intensity profile of the overlapped signal. The amplitude of the Gaussian model is equivalent to the intensity peak in the profile. The spread of the local Gaussian model for the $n^{\text {th }}$ intensity profile is defined by $\frac{\sigma \cdot h}{N}$ where $\sigma$ is the standard deviation of the intensity of voxels of the whole overlapped signal, $h_{n}$ is the normalized height of the corresponding intensity peak and $N$ is the number of intensity peaks present in the spot which has one or more signals in it (Noordmans and Smeulders., 1998).

Fig. 6, is a diagrammatic representation of the intensity profile of a two overlapping signals (shown in 1-D) and a noise artifact. The magnitude of the intensity peak $I_{n}$ of the $n^{\text {th }}$ peak is calculated from the profile plot. The local Gaussian spread $\sigma_{n}$ of the $n^{\text {th }}$ intensity peak is calculated as explained earlier. Then the Gaussian model of $n^{\text {th }}$ peak can be given as,

$$
G_{n}=I_{n} \cdot \exp \left\{\frac{x^{2}+y^{2}+z^{2}}{\sigma_{n}^{2}}\right\}
$$

The feature vector of each local Gaussian fitted profile is checked for the validity of the model to be a FISH signal. The weighted decision vector of each locally fitted Gaussian model is compared with the ideal decision vector $\vec{F}$. The object with multiple intensity peaks is considered as constituting as many FISH signals as there are peaks whose Guassian fit model decision vectors are comparable to the ideal decision vector $\vec{F}$ i.e. the error $e_{i}$ is within threshold. After separating all the overlapped signals, the FISH signal channel is again subject to 3-D component labeling as explained earlier. In the second component labeling, the labels given to FISH signals are also made to containing the label of the cell nucleus in which it is located. Signals are labeled separately for each cell nucleus and the maximum number of labels used also gives the number of FISH signals in the cell.
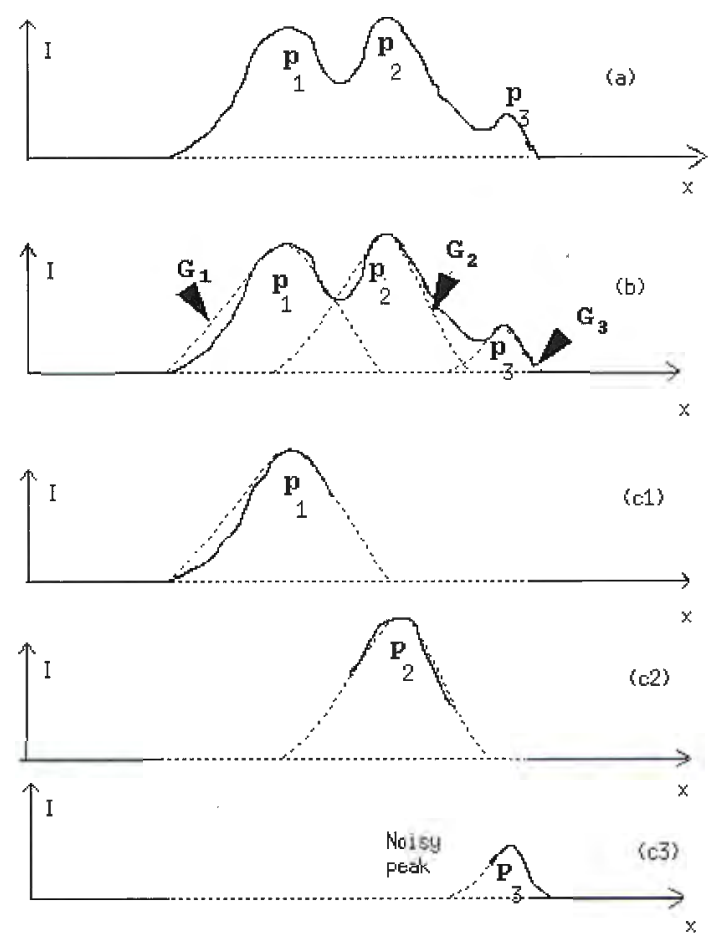

Fig. 6. Schematic diagram in 1-D of characterizing procedure for overlapping signals. (a) Original intensity profile of the spot having one or more signal peak(s) in it, (b) Approximate fit of a local Gaussian model, $(c 1, c 2, c 3)$ Separated profile of the overlapped signals. After matching the macrofeature, the third profile is rejected as noise and the first two profiles are considered as two signals. 


\section{RESULTS AND DISCUSSION}

In any field of study, reliable results are only achieved by undertaking experiments on a large number of data sets. In the case of FISH analyses using confocal microscopy, the visual counting of signals through successive cell slices is timeconsuming and fatiguing. Moreover visual counting is not always reproducible. Fig. 7 shows a sequence of image slices of the FISH signal channel after noise reduction and overlaying the border of the segmented cell nuclei. To confirm the usefulness of the automated counting of FISH signals, we have compared the visual counting results with those of automated counting on over 100 data-sets. Cells that were out of focus and not of interest to pathologist were rejected. Also the cells, which were at the border of the image were rejected since the completeness of such a cell nuclei can not be ascertained. The visual counting was done by an experienced pathologist and we consider this to be the optimal standard for all comparative study purposes. The visual counting took more than a week (including rest hours) while the automatic counting of the FISH signals in all the selected cells was completed within a period of less than an hour. Table 1 shows the result of visual counting and automatic counting. In most of the cases, we have used an integrated approach of segmentation (Adiga, 2000) of the cells for automatically marking the region of interest in the signal channel. More than $94 \%$ of the accurate comparison is obtained. From this we can conclude that the automatic counting is on par with the visual counting and the percentage of error is well within the acceptable level.

In table 2, we have compared the results obtained by using different segmentation methods and automated counting with those obtained by visual counting. Errors were sometimes observed when the FISH signal was too weak and rejected as noise and/or when a strong noise cluster was counted as a signal. The main reason for error lies in the absence of well-defined features for a FISH signal and in the fact that it is not always possible to prepare and analyze ideal specimens. Nevertheless, the percentage of error we observed lies well within the acceptable level. Although we have achieved near complete automation of FISH signal counting, our method cannot be used routinely for this purpose yet because it has only been tested on very few and similar data sets. Analytically, the methods developed are correct, but scope for improvement is ever present in the field of distinguishing noise from the signal, defining the FISH signal features, developing noise reduction methods, improving methods to identify and segment touching or overlapping signals, etc.. Furthermore, the methodology remains to be evaluated on several data sets of different types and needs to meet the approval of the large community of pathologists before it finds routine use in the future.

Table 1. Comparison of Manual signal count and automatic signal count.

\begin{tabular}{lll}
\hline Sp. No. & Visual Count & Automatic Count \\
\hline 1 & $13(5$ cells $)$ & 13 \\
2 & $10(4$ cells $)$ & 9 \\
3 & $23(9$ cells $)$ & 21 \\
4 & $31(13$ cells $)$ & 31 \\
5 & $17(6$ cells $)$ & 17 \\
6 & $8(3$ cells $)$ & 8 \\
7 & $28(11$ cells $)$ & 25 \\
8 & $23(9$ cells $)$ & 25 \\
9 & $17(8$ cells $)$ & 19 \\
10 & $33(14$ cells $)$ & 33 \\
11 & $15(6$ cells $)$ & 15 \\
12 & $21(10$ cells $)$ & 20 \\
13 & $13(4$ cells $)$ & 13 \\
14 & $18(7$ cells $)$ & 15 \\
15 & $26(11$ cells $)$ & 25 \\
16 & $9(4$ cells $)$ & 9 \\
\hline
\end{tabular}

Table 2. Comparison based on the percentage of error in FISH signal count based on different tissue segmentation techniques.

\begin{tabular}{lll}
\hline sl.nu. & Segmentation techniques & $\begin{array}{l}\text { Percentage } \\
\text { error }\end{array}$ \\
\hline 1 & Sobel edge detector & $38 \%$ \\
2 & LoG edge operator & $36 \%$ \\
3 & Canny edge operator & $28 \%$ \\
4 & Layered Segmentation & $13 \%$ \\
5 & Active contour models & $6 \%$ \\
6 & Active surface models & $9 \%$ \\
7 & Seeded Volume Growing & $16 \%$ \\
8 & Successive pealing-thickening & $8 \%$ \\
9 & 3-D watershed & $23 \%$ \\
10 & 3-D watershed with merging & $11 \%$ \\
11 & Integrated approach & $6 \%$ \\
\hline
\end{tabular}




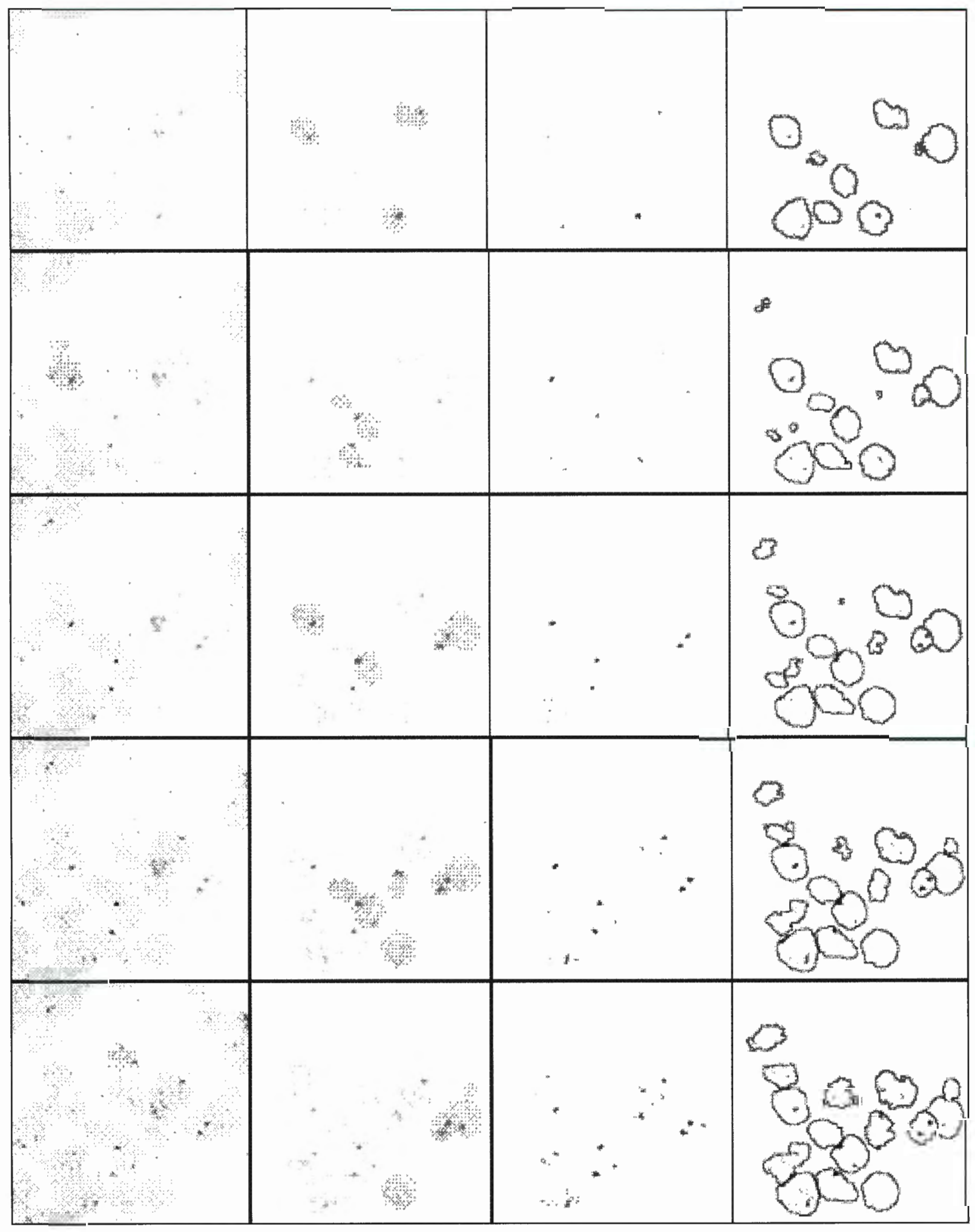




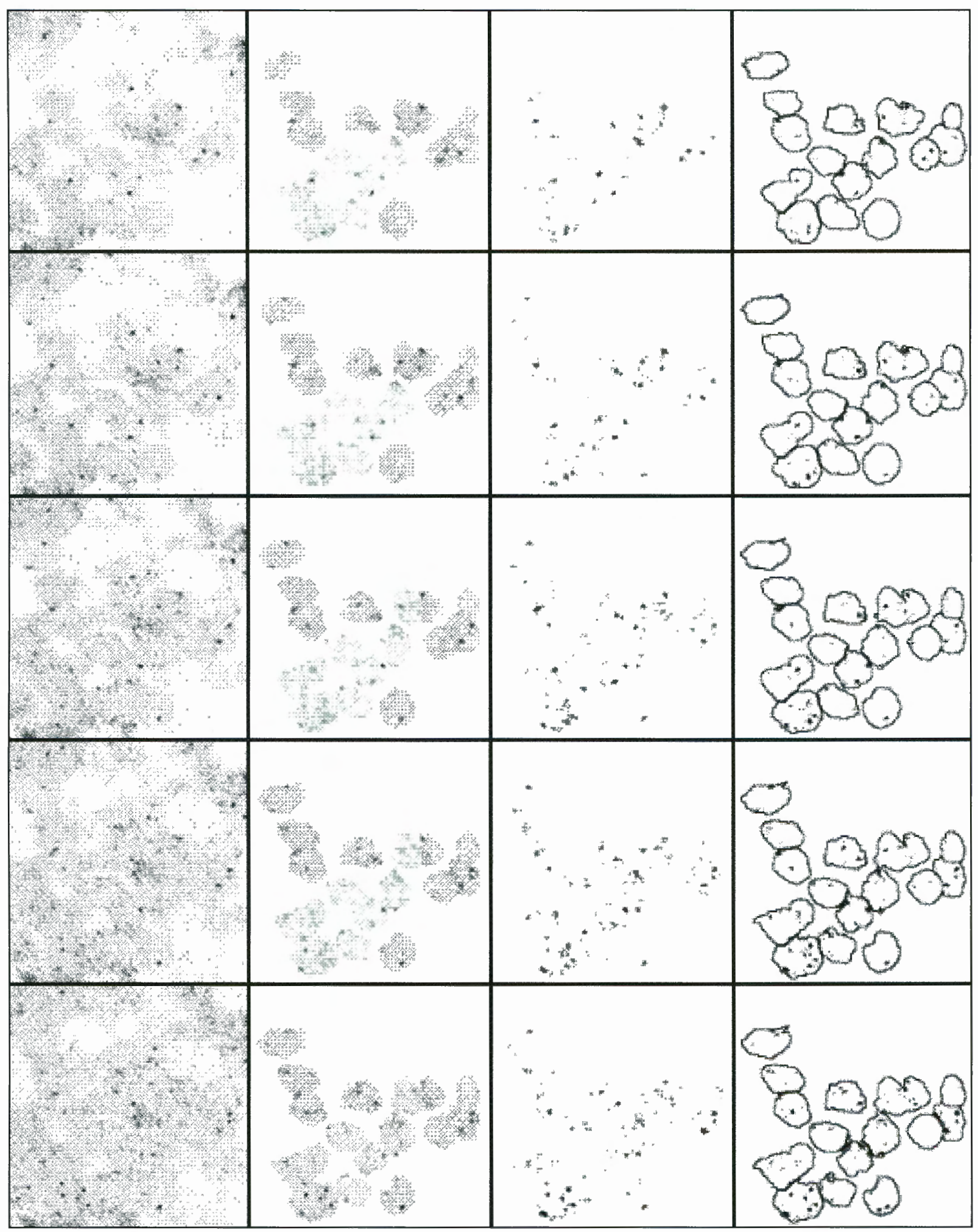

Fig. 7. Sequence of image slices of the FISH signal channel after noise reduction and over laying the border of the segmented cell nuclei. Image slices 3 to 13 are shown. Pictures are scaled to 50\% of the original size $(256 \times 256)$. 


\section{ACKNOWLEDGMENTS}

We would like to thank all the scientists of Biomedical Image Analysis Division, Institute of Pathology, GSF, Munich, Germany for providing the data sets. The work was partly supported under the project Indo-German collaboration of Bio-medical research sponsored by International Beuro, Germany and ICMR, India. We thank both the organisations for their support.

\section{REFERENCES}

Adiga PSU, Chaudhuri BB (1998a). An efficient cell segmentation tool for confocal microscopy tissue images for quantitative evaluation of FISH signals. Int $\mathrm{J}$ of Microscopy Research and Technique 43:1-20.

Adiga PSU, Chaudhuri BB (2000a). Deformable models for segmentation of CLSM images and its application in FISH signal analysis. J. Analytical Cellular Pathology, 18:211-5.

Adiga PSU, Chaudhuri BB (2000b) Region based techniques for the segmentation of volumetric histopathological images obtained using confocal microscope, J. of Computer Methods and Programs in Biomedicine, 61:23-47.

Adiga PSU and Chaudhuri BB (2000c). An efficient method based on watershed and rule based merging for segmentation of 3-D histo-pathological images. Accepted for publication in Pattern Recognition Journal.

Adiga PSU (2000). An Integrated approach for segmentation of 3-D Confocal Images of a tissue specimen. Accepted in J. of Microscopy Research Techniques.

Aubele M, Zitzelberger H, Szucs S, Werner M, Brasselman H, Hutzler P, Rodenacker K, Lehmann L, Minkus G, Hofler H (1996). Comparative FISH analysis of numerical chromosome and abnormalities in $5 \mu \mathrm{m}$ and $15 \mu \mathrm{m}$ paraffin embedded tissue sections from prostate carcinoma. Int. J. of Histochemistry Cell Biology, 107:121-6.

Babu VR, Miles BJ, Cerny JC, Weiss L, van Dyke DL (1990). Cytogenetic study of four cancers of prostate. Cancer Genetics Cytogenetics 48:83-7.

Baretton GB, Valina C, Vogt T, Schneiderbanger K, Diebold J, Lohrs U (1994). Interphase cytogenetic analysis of prostatic carcinomas by use of nonisotopic in situ hybridization. Cancer Research. 54:4472-80.

Dhingra K, Sneige N, Pandita TK, Johnson DA, Lee JS, Emami K, Hortobagyi GN, Hittleman WN (1994). Quantitative analysis of chromosomes in situ hybridization signals in paraffin embedded tissue sections. Cytometry 16:100-12.

Henke RP, Kruger E, Ayhan N, Hubner D, Hammerer P (1994). Frequency and distribution of numerical chromosomal aberrations in prostate cancer. Human Pathology 25:476-84.

Johnson GD, de Nogueira AGM (1981). A simple method of reducing fading of immuno-fluorescence during microscopy. Immunology Methods, 43:349-50.

Noordmans HJ, Smeulders AWM., (1998). Detection and characterization of isolated and overlapping spots. Computer Vision Graphics and Image Understanding, 70:23-5

Rodenacker K., Aubele M., Hutzler P. Adiga UPS., (1997). Groping for quantitative 3d image analysis : an approach to quantitative evaluation of fluorescence in situ hybridization in thick tissue sections of prostate carcinoma. Analytical Cellular Pathology 15:19-29.

Roysam B, Bhattacharya AK, Srinivas C, Szarowski DH, and Turner JN (1995). Unsupervised noise removal algorithms for 3-D confocal fluorescence microscopy. In: Biomedical Image Processing and ThreeDimensional Microscopy Acharya RS, Cogswell CJ and Goldgof DB eds. SPIE Proceedings, 16660:250-61.

Webb RH, Dorey CK (1995). The pixilated Image, In: Handbook of Biological Confocal Microscopy Pawley JB eds. Plenum Press, NewYork, 1995. 